\title{
CLUSTERING OF WALL GEOMETRY FROM UNSTRUCTURED POINT CLOUDS
}

\author{
M. Bassier, and M. Vergauwen \\ Dept. of Civil Engineering, TC Construction - Geomatics \\ KU Leuven - Faculty of Engineering Technology \\ Ghent, Belgium \\ (maarten.bassier, maarten.vergauwen)@kuleuven.be
}

Commission II

KEY WORDS: Building Information Modeling, Clustering, Wall, Building, Point Clouds

\begin{abstract}
:
The automated reconstruction of Building Information Modeling (BIM) objects from point cloud data is still ongoing research. A key aspect is retrieving the proper observations for each object. After segmenting and classifying the initial point cloud, the labeled segments should be clustered according to their respective objects. However, this procedure is challenging due to noise, occlusions and the associativity between different objects. This is especially important for wall geometry as it forms the basis for further BIM reconstruction.

In this work, a method is presented to automatically group wall segments derived from point clouds according to the proper walls of a building. More specifically, a Conditional Random Field is employed that evaluates the context of each observation in order to determine which wall it belongs too. The emphasis is on the clustering of highly associative walls as this topic currently is a gap in the body of knowledge. First a set of classified planar primitives is obtained using algorithms developed in prior work. Next, both local and contextual features are extracted based on the nearest neighbors and a number of seeds that are heuristically determined. The final wall clusters are then computed by decoding the graph and thus the most likely configuration of the observations. The experiments prove that the used method is a promising framework for wall clustering from unstructured point cloud data. Compared to a conventional region growing method, the proposed method significantly reduces the rate of false positives, resulting in better wall clusters. A key advantage of the proposed method is its capability of dealing with complex wall geometry in entire buildings opposed to the presented methods in current literature.
\end{abstract}

\section{INTRODUCTION}

As-built Building Information Modeling (BIM) models are a widely researched topic. These models reflect the state of the building up to as-built conditions and are used for quality control, quantity take-offs, maintenance and project planning (Volk et al., 2014, Patraucean et al., 2015). An as-built model is obtained by updating an existing as-design model of the structure or by reverse engineering it from measurements taken on the site. This research focuses on the creation of a BIM without a prior model since few buildings currently have a model. A key aspect in the reconstruction process is the association of the observations with the different walls in the structure. Currently, these objects are created by manually designing them based on point cloud data acquired from the built structure. However, this process is labor intensive and error prone. The retrieval of the walls is especially important since this geometry forms the basis for other objects.

Automated reconstruction approaches focus on the unsupervised processing of point clouds. The interpretation of this data is challenging due to the number of points, noise and the complexity of the structure (Tang et al., 2010). Also, most point clouds are acquired with remote sensing techniques which are bound to Lineof-Sight $(\mathrm{LoS})$. As a result, crucial parts of the structure are occluded due to clutter or inaccessible areas. Reasoning algorithms make assumptions about these zones which are prone to misinterpretation.

The emphasis of this work is on the clustering of walls from large unstructured point clouds of buildings. More specifically, we

*Corresponding author look to group wall observations on object level for the purpose of BIM reconstruction. The proposed method is able to properly detect and cluster wall geometry even in highly cluttered and noisy environments. Also, our approach deals with highly associative wall geometry as it operates directly on the $3 \mathrm{D}$ point cloud itself and is designed for multi-storey buildings.

The remainder of this work is structured as follows. The background and related work is presented in Section 2. In Section 3. the methodology is presented. The test design and experimental results are proposed in Section 4. Finally, the conclusions are presented in Section 6.

\section{BACKGROUND \& RELATED WORK}

The automated procedure of creating BIM objects from point cloud data commonly consists of the following steps (Nguyen and Le, 2013). First, the data is preprocessed for efficiency. In 2D methods, the point cloud is represented as a set of raster images consisting of a slice of the data or other information (Landrieu et al., 2017a, Anagnostopoulos et al., 2016). In 3D methods, the point cloud is restructured as a voxel octree which allows efficient neighborhood searches (Vo et al., 2015)(Fig. 1left). After the preprocessing, the data is segmented. A set of primitives is detected that replaces the point representation with the purpose of data reduction. Typically, lines are used in $2 \mathrm{D}$ methods and planes or cylinders are used in 3D methods (Vo et al., 2015, Lin et al., 2015, Fan et al., 2017, Vosselman and Rottensteiner, 2017). Next, the segments are classified by reasoning frameworks exploiting local and contextual information (Fig. 1mid). Class labels such as floors and walls are computed for each segment by using heuristics or machine learning techniques (Bassier et al., 2016, Wolf 

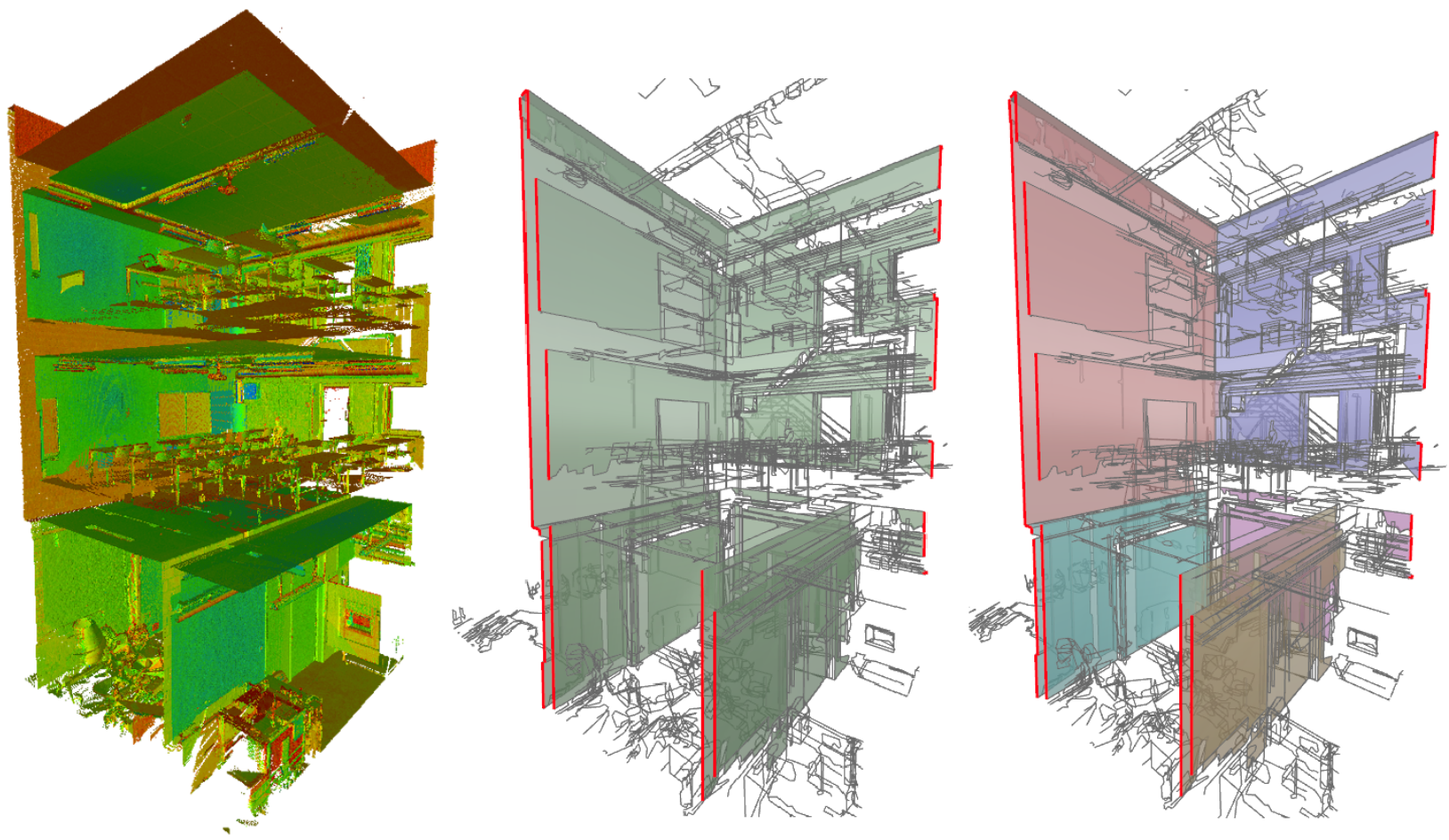

Figure 1: Overview of the different steps in the data association workflow: unstructured point cloud (left), extracted classified planar wall segments (mid) and the grouped wall observations per wall (right).

et al., 2015, Xiong et al., 2013, Nikoohemat et al., 2017). The resulting labeled wall segments are processed by a second reasoning framework to merge segments belonging to the same wall (Fig. 1right). Finally, these groups serve as input for the application specific reconstruction algorithms.

Several researchers have proposed methods for the clustering of wall geometry. Popular approaches include region growing, model based methods, graphs and machine learning techniques (Nguyen and Le, 2013). Most researchers employ a simple box definition for the wall clustering. Moreover, the majority of methods only considers one side of the wall which reduces the problem of wall detection to a plane segmentation paradigm (Mura et al., 2014). For instance, Oesau et al. (Oesau et al., 2014) assume collinearity of the segments of a wall face. They employ a Hough transform to extract all the points on the wall face in a 2D slice of the point cloud. Similar results are presented by Previtali et al. (Previtali et al., 2014), Budroni et al. (Budroni and Böhm, 2010), Valero et al. (Valero et al., 2012), Nikoohemat et al. (Nikoohemat et al., 2017) and Adan et al. (Adan and Huber, 2011). Typically, these methods prove successful in scenarios where wall detailing and complex wall geometry is not an issue.

Some researchers do actually cluster both sides of a wall into a box or rectangle. For instance, Mura et al. (Mura et al., 2014) project their detected wall segments onto a 2D plane and cluster them accordingly. A first directional clustering yields the main orientation of the walls. For every orientation, a 1D mean-shift clustering (Furukawa et al., 2009) is performed which identifies all possible offsets of parallel wall segments of that orientation. Finally, they compute a representative line for each wall cluster in 2D that is then used in the reconstruction step. Ochmann et al. (Ochmann et al., 2016) on the other hand use heuristics to cluster parallel and coplanar wall segments. Each potential pair of wall surface lines fulfilling certain constraints is considered as the two opposite surfaces of a wall separating adjacent rooms. The segments are then clustered by evaluating the overlap and the orientation of the candidates. Aside from heuristics, model based methods are also used. Recent variants of the RANSAC algorithm (Derpanis, 2010) are extended to not just fit simplistic models such as lines or planes but match entire walls. For instance, Oesau et al. (Oesau et al., 2014) employ a two-line hypothesis created by RANSAC to detect corner configurations for their walls. Similar to the single faced clustering, the majority does not consider the grouping of more complex wall geometry or highly associative walls.

Connected components Currently there is few research on the clustering of wall segments for more complex walls. However, several methods used in similar clustering tasks are also of interest. For instance, Ikehata et al. (Ikehata et al., 2015) cluster rooms based on k-medoids and heuristic segment clustering. They restructure their room observations in a raster image and use selective seeds to determine which room an observation belongs too. Armeni et al. (Armeni et al., 2016) introduce the idea of connected component graphs. They divide the initial graph into a set of subgraphs or connected component graphs by removing invalid edges based on some validation criterion. This lowers the combination complexity and also avoids misclustering. Armeni et al. use this method in combination with region growing to cluster room segments. In case a connection to a neighboring room segment contains a wall density signature, that neighbor is not considered. Other implementations focus on the clustering of roof objects. He. et al. (He et al., 2018) use connected component graphs to detect all the roof observations belonging to a single building. The concept of component graphs is a promising technique for complex wall clustering. In our implementation, we use a similar structure so solve the wall clustering. Machine learning or heuristic methods can be used to remove invalid edges from the graph after which the remaining linked observations can be clustered. For instance, Gilani et al. (Gilani et al., 2016) use connected component graphs to cluster line segments of roof edges in aerial lidar applications. Alternatively, Mura et al. (Mura et al., 2016) propose the use of structural paths for room segment clustering. They perform region growing in a adjacency matrix based 

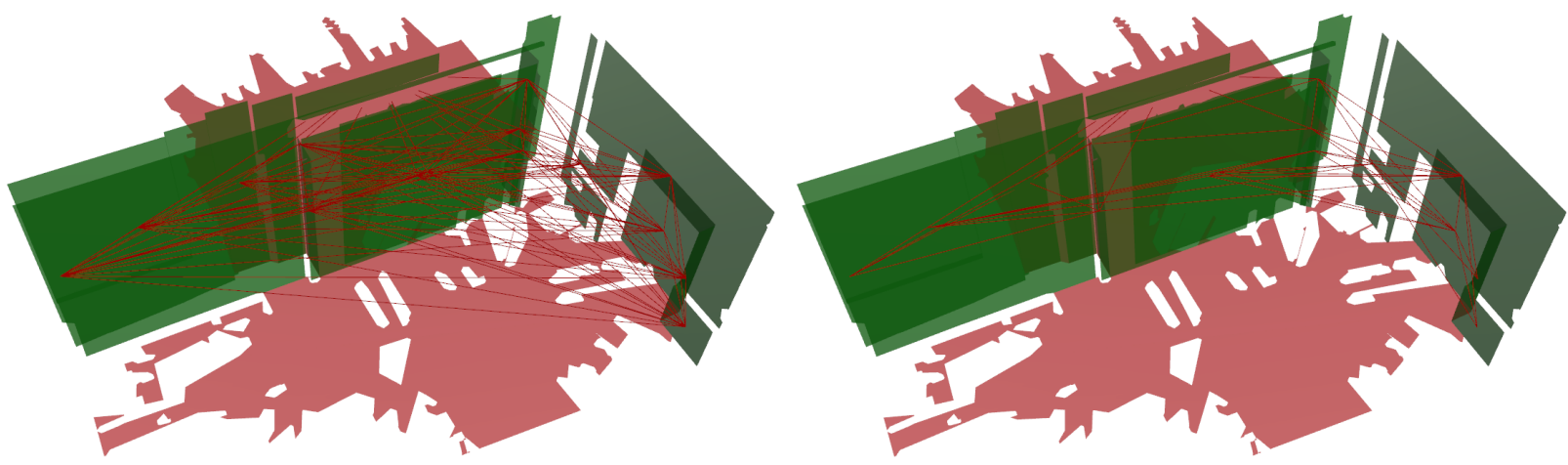

(a) Representation of the fully connected graph based on the eu- (b) Representation of the potential edges matching one of the clidean distance between nearest neighbors. three distance criteria.
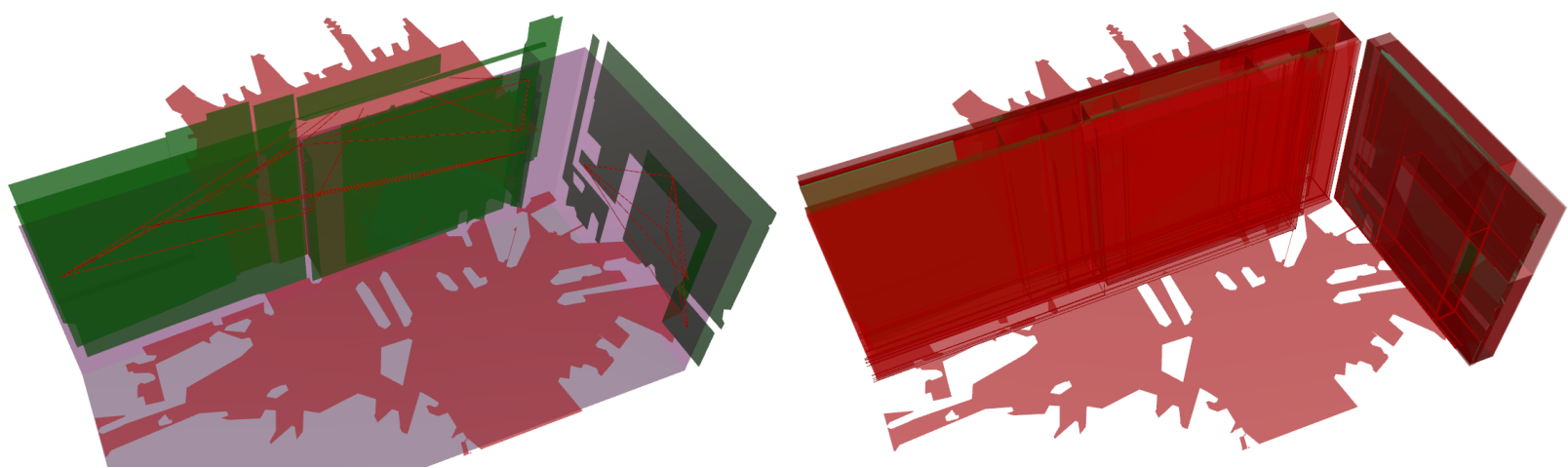

(c) Representation of the remaining edges after passing the edge (d) Representation of the remaining edges after passing the validation criterium based on the intersections with the partial bounding box validation criterium based on the dimensions of room information.

the bounding box of $s_{i}$ and $s_{j}$.
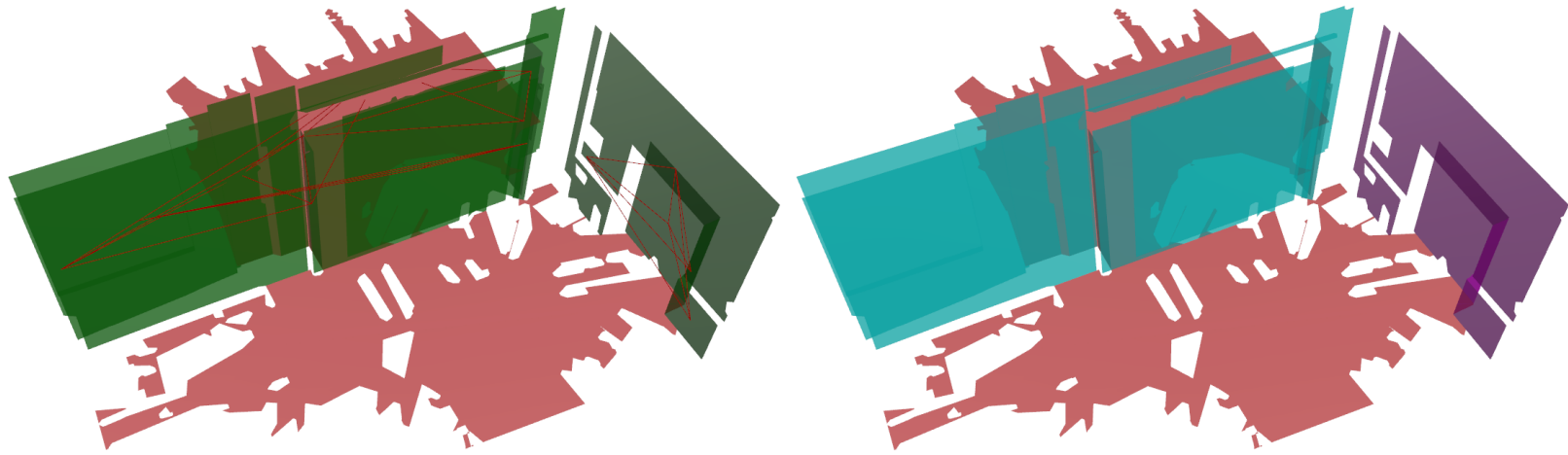

(e) Representation of the connected component graphs based on (f) Representation of the clustered wall segments passing the valthe remaining edges.

idation test or subsequent clustering.

Figure 2: Workflow of the graph based wall segments clustering depicting the consecutive steps. The wall segments are shown in green, floor segments in red and graph edges in bordeaux. 
on pairwise relations. Given the connectivity of the graph, they iteratively evaluate the edges for one of $k$ predefined connections. The result of their method is a set of patches enclosing the target space. In our research, we opt for the use of machine learning techniques in connected component graphs since the simultaneous evaluation of all observations is expected to yield better results than the one-sided stopping criteria of for instance region growing.

A promising approach for simultaneous segmentation and clustering which we adopt is the use of Conditional Random Fields (CRFs) (Sutton and Mccallum, 2011). Given the graph with a set of node and edge features, a best fit configuration can be computed by decoding the network. This can be achieved by using graph cuts (Strom et al., 2010, Turner and Zakhor, 2014, Pordel and Hellström, 2015), loopy belief propagation (Landrieu et al., 2017b, Landrieu et al., 2017a, Vosselman and Rottensteiner, 2017) or other approximation techniques. The key to this technique is the proper seeding to determine the number of walls which can prove difficult with wall segments. While the parameter estimation of these models can be challenging, we believe it a promising approach to cluster complex scenarios and highly associative wall configurations with high recall and precision.

\section{METHODOLOGY}

In this paper, a clustering algorithm is proposed that groups the planar wall segments into wall clusters. The procedure consists of the following steps. First, the point cloud data is segmented and semantically labeled using prior algorithms (Bassier et al., 2018). Next, a initial segmentation is performed to create a set of connected component graphs and to establish a set of seeds. In a final stage, the observations within each component are clustered according to the individual walls given the seed segments. The consecutive steps are discussed in detail in the following paragraphs.

Data preprocessing Prior to the clustering, the data is segmented and classified. First, the unstructured point cloud is represented as a voxel octree after which planar patches are extracted from the data as presented in our previous research (Bassier et al., 2017). Next, the planar patches are subjected to a reasoning framework that computes class labels for each patch. A pretrained Random Forests model is used for the classification (Bassier et al., 2018). The result is a set of labeled segments that replaces the point cloud representation of the building.

The connected component graph is constructed as follows. First, a densely connected graph $G(N, E)$ is defined with a set of nodes $N$ representing the wall segments $s$ and a set of edges $E$ representing the connections between neighboring segments (Fig. 2 a). The adjacency matrix is constructed based on the euclidean distance between the boundaries of the segments. Each $n$ is connected to its $k$ nearest neighbors. Each edge $e_{i j}$ is conditioned to comply with at least one of the following criteria (Eq. 1): The absolute distance between boundaries, the distance between coplanar segments and the distance between parallel segments given the overlap between $s_{i}$ and $s_{j^{\prime}}$ with $s_{j^{\prime}}$ the projected geometry of $s_{j}$ onto $s_{i}$ along $\overrightarrow{n_{i}}$ (Fig. 2 b).

$$
e_{i j} \in G:\left\{\begin{array}{l}
\left\|s_{i}, s_{j}\right\| \leq t_{d} \\
\overrightarrow{n_{i}} \cdot \overrightarrow{n_{j}} \geq t_{\theta} \quad \& \quad \overrightarrow{n_{i}} \cdot\left(\overrightarrow{c_{s_{i}}}-\overrightarrow{c_{s_{j}}}\right) \leq t_{\text {copl }} \quad \& \\
\quad\left\|s_{i}, s_{j}\right\| \leq t_{d, \text { copl }} \\
\overrightarrow{n_{i}} \cdot \overrightarrow{n_{j}} \geq t_{\theta} \quad \& \quad\left\|s_{i}, s_{j^{\prime}}\right\| \leq t_{\text {par }}
\end{array}\right.
$$

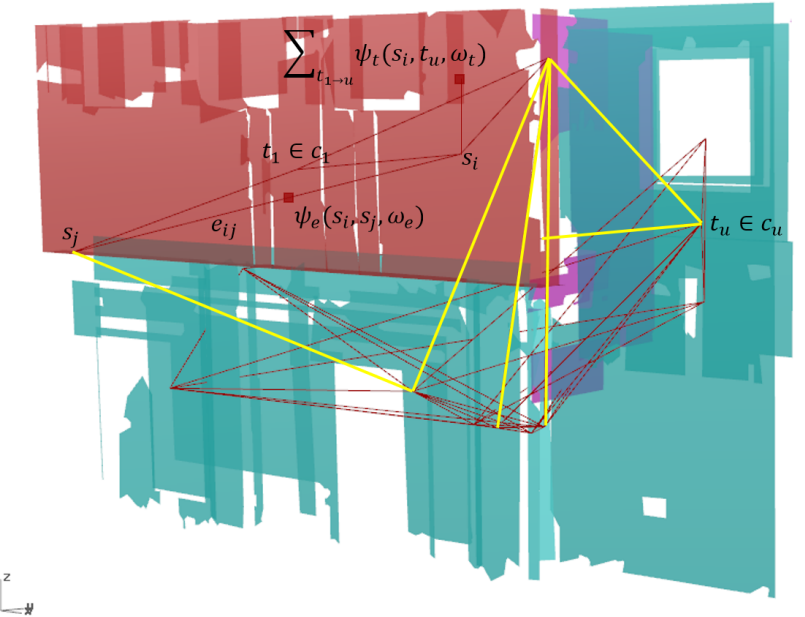

Figure 3: Illustration of the clustering using Conditional Random Fields within a connected component graph: given the seeds $t_{1 \rightarrow u}$ from the region growing, one of $u$ labels is computed for each $s$ given the factor graph with the edge and node potentials. The three clusters are depicted in red, cyan and purple, the edges are shown in red and the edges between clusters are shown in yellow.

Subsequently, the valid edges are subjected to the following two validation criteria. An edge $e_{i j}$ may not intersect with a set of reference surfaces. This set is composed of both wall segments as well as basic room information. For this evaluation, the ceiling geometry is extruded to the height of the underlying floors to form enclosed spaces (Fig. 2 c). The boundaries of these volumes along with the wall segments are tested for collisions with the edges and intersecting edges are removed. The second criterion is a model based evaluation that tests the potential wall thickness (Fig. 2 d). A bounding box is constructed between every connected $s_{i}$ and $s_{j}$ and the thickness is tested along the normal of the largest wall segment. If the thickness exceeds a threshold $t_{b}$, the edge is considered invalid. By removing the invalid links from the network, a set of connected component graphs $G^{\prime}$ is created that each contains a number of connected segments (Fig. 2 e).

Clustering In this work, the clustering paradigm is considered a classification task that can be solved with supervised pattern recognition techniques. More specifically, we propose the use of Conditional Random Fields (CRF) as discussed in the related work. A major advantage of this technique is that every seed is evaluated simultaneously so that the clustering is not dependent on heuristic stopping criteria as is the case with region growing methods. This is expected to result in a more balanced clustering where each $s$ is assigned to the best fit $c$ instead of the cluster with the largest seed. The objective is to find the most optimal labeling of the set of nodes represented by $s$ that can take one of $c \in u=\left\{c_{1}, c_{2}, \ldots, c_{u}\right\}$ states. This is performed by computing the a posteriori probability distribution of the states over the nodes in the graph. In order to do so, the graph is restructured as a factor graph (Fig. 3). Depending on the number of output clusters in the component graph, a set of unary and pairwise potentials is established for each node and edge. The set $E$ of edges $e_{i j}$ in the network is given non-negative weights along with a set of feature vectors. Additionally, we replace the nodes that function as the seeds by terminal nodes similar to the graph structure used in graph cuts. These nodes $t$ take one of $u$ fixed states since they serve as seeds. The labeling is performed by maximizing the probability over the network. Each of the above steps, including the seeding for the number of outputs, the edge potential initial- 


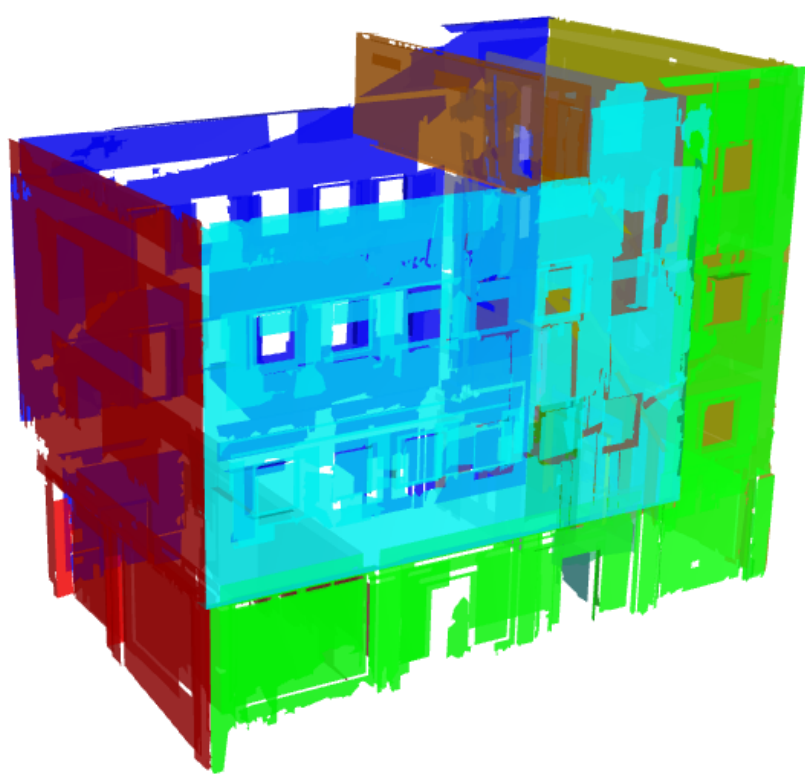

(a) Representation of the ground truth data with each wall in a seperate color.

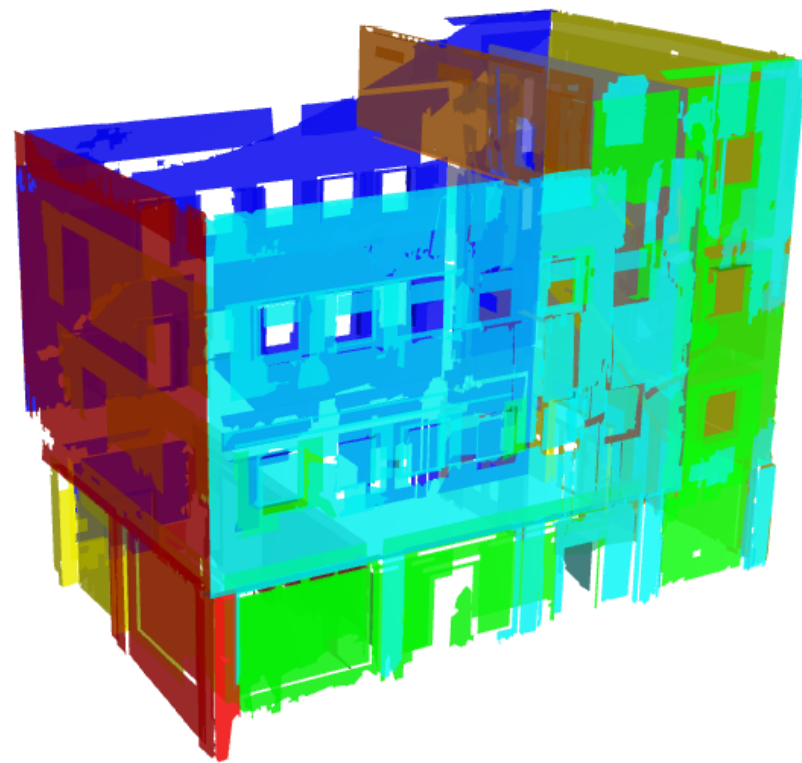

(b) Representation of the clustered wall segments using Conditional Random Fields (CRF).

Figure 4: Test data set of a school facility on the Campus.

ization and the labeling are discussed in the paragraphs below.

Seeding It is stated that while the clustering of region growing itself is likely to be erroneous, at least a part of each wall is represented in a region. Therefore, we use the largest segment $s_{l}$ in each region as the seeds for the graph. The region growing algorithm operates as follows. Iteratively, the neighbors $s_{i}$ of $s_{l}$ are evaluated. Starting from the largest neighbor, a bounding box criterion similar to the initial graph definition is evaluated. However, instead of computing the bounding box of only $s_{l}$ and $s_{i}$, all the $s$ in the current $c$ are evaluated along the average $\overrightarrow{n_{c}}$ weighted by the percentual surface areas of $s \in c_{u}$. Given this bounding box, $s_{i}$ is merged with the cluster if the total bounding box thickness does not exceed $t_{b}$. This hard constraint allows for flexible growing but restricts the cluster from becoming too large. If no more members can be added to the cluster, the largest unclustered segment is selected as new seed and the process is repeated for the remaining surfaces. Additionally, the clusters are conditioned to have more than one surface or that the surface area is sufficiently large. This avoids singular surfaces that do not fit well with other segments to result in erroneous walls. The result is a set of clusters $c \in C$, with each $c$ representing the available observations of a single wall. The nodes corresponding to the seeding segments are given a fixed state and will serve as the basis for the feature extraction in the CRF.

Potentials The potentials are initialized for each edge $e_{i j}$ and node $s$ in the graph. We define both pairwise and unary potentials to determine which cluster a node belongs to. The pairwise potentials are computed given a set of edge features $f_{e}\left(s_{i}, s_{j}\right)$ and corresponding weights $\omega_{e}$. Associative values are computed for the euclidean distance between the boundaries of $s_{i}$ and $s_{j}$ and the potential bounding box thickness according to the largest segment of the two neighbors. We use exponential functions to model the information to ensure non negative associative features.

The unary potentials are initialized based on the affinity of a node to a certain cluster $c_{u}$ represented by each of the seed nodes $t_{u}$. A set of features $f_{t(1 \rightarrow u)}\left(s_{i}, t_{u}\right)$ and corresponding weights $\omega_{t}$ are defined. In this work, two feature are defined for each seed. The features include the thickness of the bounding box of $s_{i}$ and $t_{u}$ and the absolute distance between the boundaries of $s_{i}$.

Probability Estimation The a posteriori probability distribution of the Conditional Random Field is found by factorizing the graph. We define the conditional probability of the states in the network as follows (Eq. 2).

$$
\begin{array}{r}
P\left(c \mid f_{t}, f_{e}, \boldsymbol{\omega}\right)=\frac{1}{Z\left(f_{t}, f_{e}\right)} \exp \left(\sum_{s \in S, t_{1 \rightarrow u}} \omega_{t} \psi_{t}\left(f_{t_{u}}, c_{i}\right)\right. \\
\left.+\sum_{i, j \in E} \omega_{e} \psi_{e}\left(f_{e_{i, j}}, c_{i}, c_{j}\right)\right)
\end{array}
$$

where $Z\left(f_{t}, f_{e}\right)$ is the normalizing partitioning function, $\psi_{t}\left(f_{t_{u}}, c_{i}\right)$ describes the unary potentials of the nodes with relation to the seed nodes and $\psi_{e}\left(f_{e_{i, j}}, c_{i}, c_{j}\right)$ describes the pairwise potentials between neighboring nodes. The graph is decoded by maximizing the conditional probability. However, exact decoding is unfeasible in a densely connected CRF. Therefore, we approximate the decoding using a generalized iterated conditional mode algorithm as implemented by Schmidt (Schmidt, 2010). After computing the most likely configuration of the graph, the segments are clustered according to the labels. The result is a set of clustered wall segments.

\section{EXPERIMENTS}

The algorithm is tested on a school facility on the technology campus in Ghent, Belgium. It is a four-storey structure with a variety of rooms including a laboratory, two classrooms, a staircase and a maintenance room (Fig. 4). A large amount of clutter is present in the environment. Nearly 7000 surfaces were detected, 258 of which represent wall observations. A total of 14 multi-storey walls were defined for the testing (Fig. 4). $k=20$ 


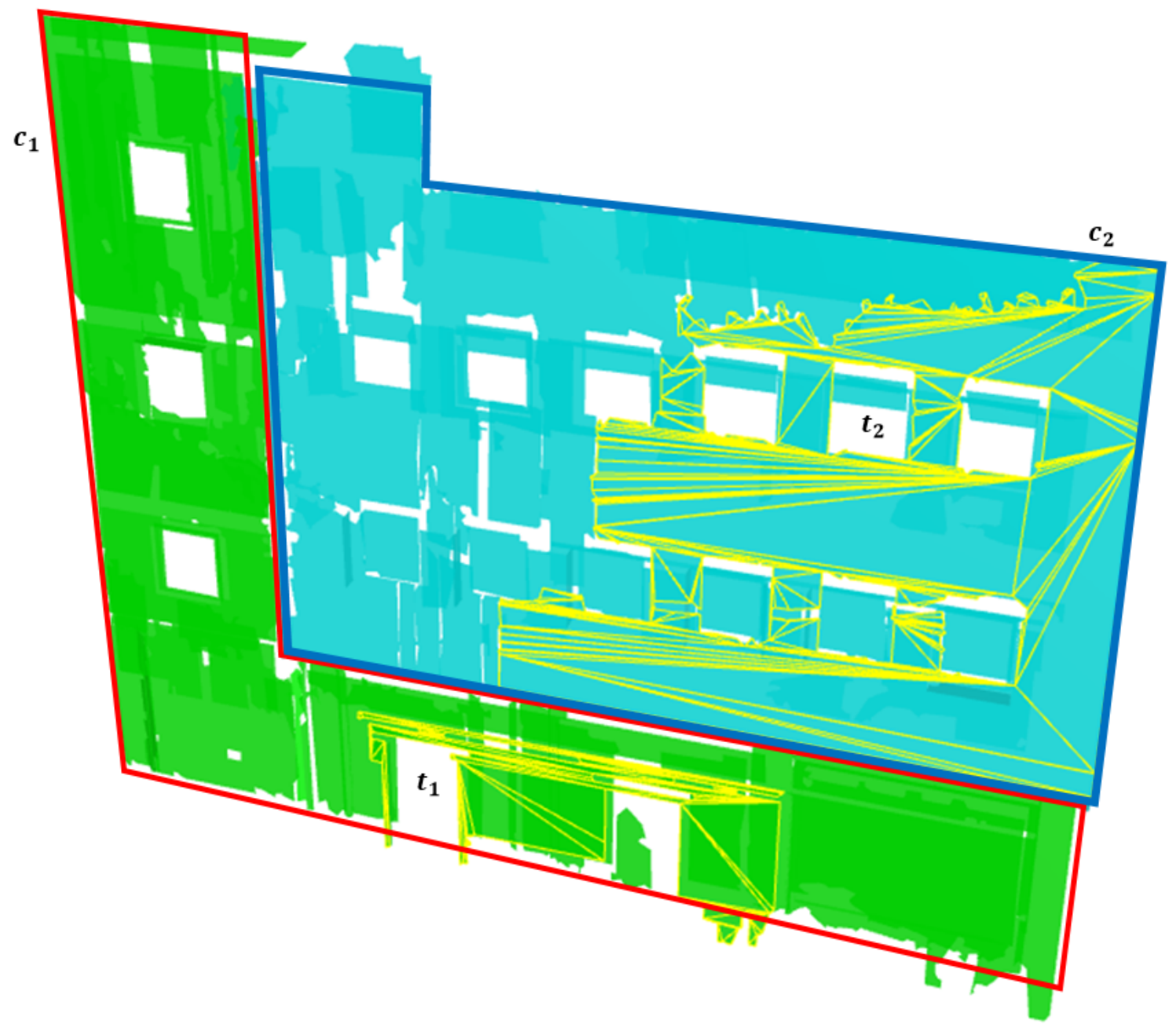

(a) Ground truth of two highly associative walls $c_{1}$ (red cluster) and $c_{2}$ (blue cluster).

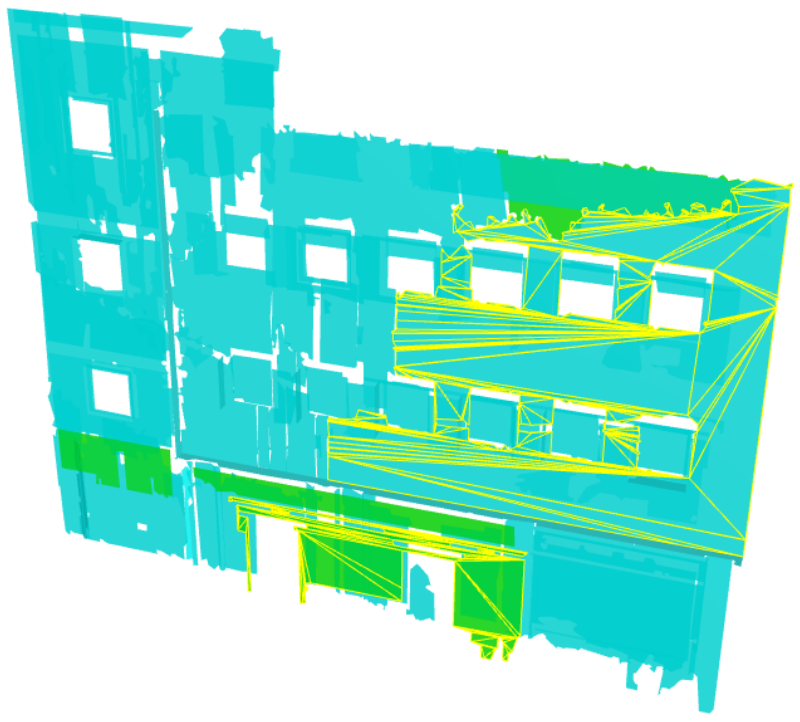

(b) Region Growing clustering.

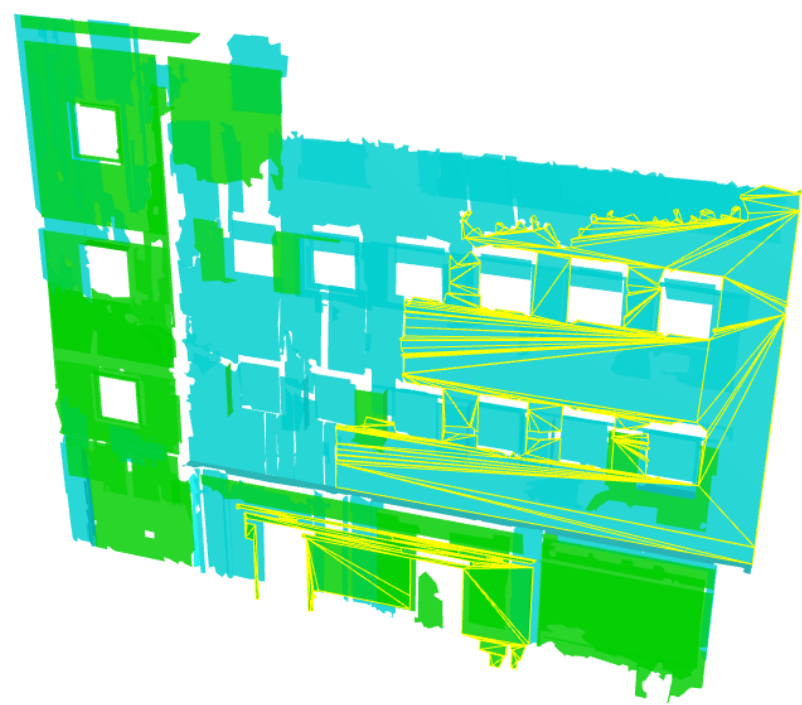

(c) Conditional Random Fields clustering.

Figure 5: Comparison between the results of the proposed CRF clustering algorithm and a conventional region growing algorithm. The seed nodes are represented in yellow and the walls are colored according to their respective clusters. 
connections were evaluated for each $s$. The following parameters were used for the region growing. The bounding box thickness $t_{b}$ was set to $0.8 \mathrm{~m}$, the absolute distance between boundaries $t_{d} \leq 0.5 m$, the coplanar distance $t_{c o p l} \leq 1 m$, the coplanar boundary distance $t_{d-\text { copl }} \leq 2 m$ and the distance $t_{p a r}$ between overlapping parallel $s$ is equal to $t_{b}$. In total, 7 connected components were detected in $6.7 \mathrm{~s}$ of which 6 components consisted of a single surface. In the validation step, 6 of the 7 components complied with the thickness criterion $t_{b}$ and thus were isolated.

The 216 segments within the remaining connected component graph were processed by the proposed Conditional Random Fields method. For the state estimation, the seeds are used that were extracted from the region growing. 7 valid seeds were detected. As unary potentials, the bounding box thickness and edge length were computed between the observations and each seed segment. The pairwise potentials are initialized based on the same features but between neighboring segments. The weights of the features was set equally over all states as there shouldn't be bias over which cluster an observation belongs to. The decoding was performed using a generalized iterated conditional mode algorithm as implemented by Schmidt (Schmidt, 2010). $74.5 \%$ of the segments were properly clustered in less than $1 s$. Together with the initial valid connected components, $78 \%$ of the wall geometry was assigned to the proper cluster. This is promising given the complexity of the structure and the variety of the geometry. However, some errors still remain. Especially smaller segments are error prone due to their high associativity with multiple seeds.

\section{COMPARISON}

For the validation, the results of the CRF method are compared to the results of a conventional region growing algorithm that was used to establish the seeds. Both methods operate with the same parameters and features. As discussed above, the main difference between these methods is the simultaneous assignment of the segments with the CRF in contrast to the one-sided stopping criteria of the region growing. As an example, the results of two highly associative walls are discussed (Fig. 5). These walls are near coplanar with several segments of $c_{1}$ surrounding $c_{2}$, have different thicknesses at different heights and have protrusions, niches and recesses. This is considered a worst case scenario where even experienced modelers would struggle to assign the segments to the proper clusters.

As discussed above, the region growing method prioritizes segments with a large surface area. Therefore, the algorithm iterates through the segments and evaluates the observations with respect to $t_{2}$. As expected, the majority of the segments comply with the validation criteria based on $c_{2}$. The remainder of the segments is assigned to $c_{1}$ leading to a severely unbalanced clustering. In the CRF method, the features between neighbors and with respect to $t_{1}$ and $t_{2}$ are evaluated simultaneously leading to a more proper clustering. It is observed that while the results of the CRF are far from perfect, it provides a significantly better clustering than the conventional region growing. It is argued that with the implementation of additional features, this clustering can be further enhanced to provide a proper clustering of at least the significant portion of each wall.

\section{CONCLUSION}

This paper presents an unsupervised method to cluster wall geometry from unstructured point clouds of buildings. A 3D approach is proposed that deals with complex multi-storey data sets.
First, the data is preprocessed by a segmentation and classification framework to drastically reduce the data and to increase the level of information. The resulting labeled segments are processed by the clustering algorithm to group the wall observations per wall object. First, an initial segmentation is performed to create a set of connected component graphs and to establish a set of seeds. In a second stage, the observations within each component are clustered according to the individual walls given the seed segments. A method based on Conditional Random Fields is proposed to perform the clustering. Both unary and pairwise features are considered encoding the associativity between neighboring segments and a set of seed nodes that are extracted from region growing. Once the state of each observation is determined, the segments are assigned to their respective clusters. The result is a set of clustered segments that correspond to the wall objects in a building.

The experiments indicates that the used method is a promising clustering framework for unstructured point cloud data. Over $78 \%$ recall is reported for the clustering of the wall segments. When comparing the proposed method to a conventional region growing method, it is revealed that the proposed method leads to a more appropriate grouping of the segments. However, some errors are still present in complex scenarios where there is confusion about which cluster a segment belongs too. Especially in highly associative scenes such as with near coplanar walls, the clustering underperforms. In future work, additional features will be implemented to enhance the clustering of these scenes.

\section{ACKNOWLEDGEMENTS}

This project has received funding from the European Research Council (ERC) under the European Union's Horizon 2020 research and innovation programme (grant agreement 779962) and the Geomatics research group of the Department of Civil Engineering, TC Construction at the KU Leuven in Belgium.

\section{REFERENCES}

Adan, A. and Huber, D., 2011. 3D Reconstruction of Interior Wall Surfaces under Occlusion and Clutter. 2011 International Conference on 3D Imaging, Modeling, Processing, Visualization and Transmission pp. 275-281.

Anagnostopoulos, I., Patraucean, V., Brilakis, I. and Vela, P., 2016. Detection of walls, floors and ceilings in point cloud data. In: Construction Research Congress 2016.

Armeni, I., Sener, O., Zamir, A. R., Jiang, H., Brilakis, I., Fischer, M. and Savarese, S., 2016. 3D Semantic Parsing of Large-Scale Indoor Spaces. Proceedings of the IEEE International Conference on Computer Vision and Pattern Recognition.

Bassier, M., M, B., Van Genechten, B. and M, V., 2017. OctreeBased Region Growing and Conditional Random Fields. The International Archives of the Photogrammetry, Remote Sensing and Spatial Information Sciences, Volume XLII-2/W8, 2017 5th International Workshop LowCost 3D - Sensors, Algorithms, Applications XLII(November), pp. 28-29.

Bassier, M., Van Genechten, B., Vergauwen, M., Genechten, B. V. and Vergauwen, M., 2018. Classification of sensor independent point cloud data of building objects using random forests. Journal of Building Engineering (April), pp. 1-10.

Bassier, M., Vergauwen, M. and Van Genechten, B., 2016. Automated Semantic Labelling of 3D Vector Models for Scan-toBIM. 4th Annual International Conference on Architecture and Civil Engineering (ACE 2016) (April), pp. 93-100. 
Budroni, A. and Böhm, J., 2010. Automatic 3d Modelling Of Indoor Manhattan-world Scenes From Laser Data. ISPRS Commission V Mid-Term Symposium: Close Range Image Measurement Techniques XXXVIII, pp. 115-120.

Derpanis, K. G., 2010. Overview of the RANSAC Algorithm. Image Rochester NY 4(1), pp. 2-3.

Fan, Y., Wang, M., Geng, N., He, D., Chang, J. and Zhang, J. J., 2017. A self-adaptive segmentation method for a point cloud. Visual Computer pp. 1-15.

Frey, B. and MacKay, D. J. C., 1998. A Revolution: Belief Propagation in Graphs With Cycles. Advances in neural information processing systems.

Furukawa, Y., Curless, B., Seitz, S. M. and Szeliski, R., 2009. Reconstructing building interiors from images. 2009 IEEE 12th International Conference on Computer Vision (Iccv), pp. 80-87.

Gilani, S. A. N., Awrangjeb, M. and Lu, G., 2016. An automatic building extraction and regularisation technique using LiDAR point cloud data and orthoimage. Remote Sensing 8(3), pp. 1-27.

He, X., Zhang, X. and Xin, Q., 2018. Recognition of building group patterns in topographic maps based on graph partitioning and random forest. ISPRS Journal of Photogrammetry and Remote Sensing 136, pp. 26-40.

Ikehata, S., Yang, H. and Furukawa, Y., 2015. Structured Indoor Modeling. Proceedings of the IEEE International Conference on Computer Vision p. 1540012.

Landrieu, L., Mallet, C. and Weinmann, M., 2017a. Comparison of belief propagation and graph-cut approaches for contextual classification of 3D lidar point cloud data. IGARSS'2017.

Landrieu, L., Raguet, H., Vallet, B., Mallet, C. and Weinmann, M., 2017b. A structured regularization framework for spatially smoothing semantic labelings of 3D point clouds. ISPRS Journal of Photogrammetry and Remote Sensing 132, pp. 102-118.

Lin, Y., Wang, C., Cheng, J., Chen, B., Jia, F., Chen, Z. and Li, J., 2015. Line segment extraction for large scale unorganized point clouds. ISPRS Journal of Photogrammetry and Remote Sensing 102(March), pp. 172-183.

Mura, C., Mattausch, O. and Pajarola, R., 2016. Piecewise-planar Reconstruction of Multi-room Interiors with Arbitrary Wall Arrangements. Computer Graphics Forum 35(7), pp. 179-188.

Mura, C., Mattausch, O., Jaspe Villanueva, A., Gobbetti, E. and Pajarola, R., 2014. Automatic room detection and reconstruction in cluttered indoor environments with complex room layouts. Computers \& Graphics 44, pp. 20-32.

Nguyen, A. and Le, B., 2013. 3D point cloud segmentation : A survey 3D. 2013 6th IEEE Conference on. IEEE (November), pp. 225-230.

Nikoohemat, S., Peter, M., Oude Elberink, S. and Vosselman, G., 2017. Exploiting Indoor Mobile Laser Scanner Trajectories for Semantic Interpretation of Point Clouds. ISPRS Annals of Photogrammetry, Remote Sensing and Spatial Information Sciences IV-2/W4(September), pp. 355-362.

Ochmann, S., Vock, R., Wessel, R. and Klein, R., 2016. Automatic reconstruction of parametric building models from indoor point clouds. Computers \& Graphics 54, pp. 94-103.

Oesau, S., Lafarge, F. and Alliez, P., 2014. Indoor scene reconstruction using feature sensitive primitive extraction and graphcut. ISPRS Journal of Photogrammetry and Remote Sensing 90, pp. $68-82$.
Patraucean, V., Armeni, I., Nahangi, M., Yeung, J., Brilakis, I. and Haas, C., 2015. State of research in automatic as-built modelling. Advanced Engineering Informatics 29, pp. 162-171.

Pordel, M. and Hellström, T., 2015. Semi-Automatic Image Labelling Using Depth Information. computers pp. 142-154.

Previtali, M., Barazzetti, L., Brumana, R. and Scaioni, M., 2014. Towards automatic indoor reconstruction of cluttered building rooms from point clouds. ISPRS Annals of Photogrammetry, Remote Sensing and Spatial Information Sciences II-5(June), pp. 281-288.

Schmidt, M., 2010. Graphical Model Structure Learning with 1 -Regularization. PhD thesis, University of British Columbia.

Strom, J., Richardson, A. and Olson, E., 2010. Graph-based segmentation for colored 3D laser point clouds. IEEE/RSJ $2010 \mathrm{In}$ ternational Conference on Intelligent Robots and Systems, IROS 2010 - Conference Proceedings pp. 2131-2136.

Sutton, C. and Mccallum, A., 2011. An Introduction to Conditional Random Fields. Foundations and Trends in Machine Learning 4(4), pp. 267-373.

Tang, P., Huber, D., Akinci, B., Lipman, R. and Lytle, A., 2010. Automatic reconstruction of as-built building information models from laser-scanned point clouds: A review of related techniques. Automation in Construction 19(7), pp. 829-843.

Turner, E. and Zakhor, A., 2014. Floor Plan Generation and Room Labeling of Indoor Environments from Laser Range Data. GRAPP, International Joint Conference on Computer Vision, Imaging and Computer Graphics Theory and Applications pp. 112 .

Valero, E., Adán, A. and Cerrada, C., 2012. Automatic method for building indoor boundary models from dense point clouds collected by laser scanners. Sensors (Switzerland).

Vo, A. V., Truong-Hong, L., Laefer, D. F. and Bertolotto, M., 2015. Octree-based region growing for point cloud segmentation. ISPRS Journal of Photogrammetry and Remote Sensing 104, pp. 88-100.

Volk, R., Stengel, J. and Schultmann, F., 2014. Building Information Modeling (BIM) for existing buildings - Literature review and future needs. Automation in Construction 38, pp. 109-127.

Vosselman, G. and Rottensteiner, F., 2017. Contextual segmentbased classification of airborne laser scanner data. ISPRS Journal of Photogrammetry and Remote Sensing.

Wolf, D., Prankl, J. and Vincze, M., 2015. Fast Semantic Segmentation of 3D Point Clouds using a Dense CRF with Learned Parameters. IEEE International Conference on Robotics and Automation (ICRA).

Xiong, X., Adan, A., Akinci, B. and Huber, D., 2013. Automatic creation of semantically rich 3D building models from laser scanner data. Automation in Construction 31, pp. 325-337. 